\title{
Das Archiv der Freiherren von Thüngen Lutzischer Linie in Zeitlofs.
}

\author{
Von \\ Rudolf Freiherrn von Thüngen, \\ k. bayer. Kämmerer und Oberstleutnant a. D. ${ }^{1}$ )
}

Der Bau dieses Archives wurde im Mai 1911 begonnen, im Herbst 1911 im Rohbau und im Mai 1912 ganz vollendet. Das Gebäude steht frei im Hofe des Freiherrl. von Thüngenschen Rentamts in Zeitlofs, getrennt von den nächsten, nicht höher als zweistöckigen Gebäuden, in der Nähe der Kirche, also möglichst geschützt gegen Feuersgefahr.

Die Größenverhältnisse - - aus dem beiliegenden Plan des näheren ersichtlich -- sind dem Bedarf auf absehbare Zeit ent-

1) Dieser Beitrag, für den ich mich seinem Verfasser zu besonderem Danke verpflichtet fühle, hat mir als Redakteur dieser Zeitschrift und als Archivar eine seltene Freude bereitet, denn er lehrt uns die Geschichte eines großen Adelsarchives kennen und gibt Zeugnis für clessen hohe Wertschätzung von seiten seiner Edelherren, die sie in rorbildlicher Weise sogar zu einem eigenen Archivbau geführt hat, eine Tat, die den Fortbestand dieses Archives verbürgt und damit für die Geschichte des uralten Geschlechtes von Thüngen, aber auch für die (ieschichtc und Heimatkunde des Frankenlandes eine sehr reiche Quelle sichert. Dieser Archivneubau in Zeitlofs verdient in weitesten Kreisen bekannt zu werden, er ist um so anerkennenswerter, als er meines Wissens im Königreiche Bayern seinesgleichen nicht hat. Kein anderes bayerisches Adelsgeschlecht hat bis jetzt seinem Archive eine eigene Heimstätte gebaut. Möge das leuchtende Vorbild des Archives der Freiherren von Thüngen in Zeitlofs bei anderen Edelgeschlechtern im engeren und weiteren Vaterlande Nachahmung finden, alle aber zur Ordnung und möglichsten Sicherung ihrer Archive, der Hauptzeugen ihrer Vergangenheit, aneifern! Dr. v. Baumann. 
sprechend, da außer dem eigentlichen Archivraum von $11,20 \mathrm{~m}$ zu 7,16 m Grundfläche und 3,70 m lichter Höhe noch ein Kellerraum von gleicher Grundfläche und $2,10 \mathrm{~m}$ lichter Höhe vorhanden ist, der sich ebenfalls zur Aufbewahrung von Akten eignet. Die äußeren Maße sind: 12,30 m Länge, 8,20 m Breite und $8,20 \mathrm{~m}$ Höhe vom Erdboden bis zum First.

Die Kellermauern, aus Findlings-Sandsteinen hergestellt, sind $0,60 \mathrm{~m}$ stark, der Kellerfußboden betoniert und kanalisiert, die Kellerdecke aus I-Trägern und Beton ist $0,20 \mathrm{~m}$ stark; in den einzelnen Gewölbefeldern sind Rundeisen von $10 \mathrm{~mm}$ Durchmesser in Abständen von $0,25 \mathrm{~m}$ einbetoniert worden.

Der Archivraum selbst hat Umfassungsmauern von Backsteinen, $0,50 \mathrm{~m}$ stark, das Material der Decke ist Eisenbeton, das des Fußbodens Terrazzo, die Fenster haben Eisenrahmen und sind mit eisernen Gittern und eisernen, von innen verschließbaren Läden versichert.

Auf der Eisenbetondecke ist ein wasserdichter Zementglattstrich aufgebracht, damit bei einem eintretenden Dachstuhlbrand kein Wasser in den Archivraum eindringen kann.

Von den zwei Türen ist die äußere von der bekannten Firma Küttenbaum in Würzburg nach modernen Grundsätzen diebsund feuersicher ausgeführt. Sie ist aus Stahl und mit drei Schlössern versehen. Die innere aus Eisenblech ist einfach verschließbar.

Der hölzerne Dachstuhl ist mit Patentfalzziegeln eingedeckt.

Gegen das Aufsteigen von (irundfeuchtigkeit sind die Mauern oberhalb des Erdbodens mit starken Isolierpappen abgedeckt.

Die zwei Kellerfenster mit eisernen Rahmen sind durch Gitter noch weiter geschützt.

Die Luftzuführung erfolgt durch 12 Mauerschlitze (6 am Fußboden, 6 an der Decke), die innen und außen mit Gittern (Mäuse und Vogelnester) versehen sind.

Die Gesamtkosten des Baues betrugen fast genau $13000 \mathrm{M}$.

Die Bauausführung geschah durch heimische Maurer usw. unter der Oberaufsicht des Distriktsbaumeisters von Brückenau. Die Mauern sind beworfen; das Gebäude von einfacher Ausführung zeigt als Schmuck nur am Deckstein der Eingangstüre das Thüngensche Wappenschild und die Jahreszahl 1911. 
Das Archiv befand sich vor Errichtung des Neubaues in einem gewölbten ebenerdigen Raum des. Zeitlofser Schlosses. Die Verlegung erfolgte nicht, weil der Raum ungenügend sicher erschienen wäre, obgleich eine gewisse Gefahr in der Vereinigung mit einem bewohnten Gebäude nicht verkannt wurde, sondern hauptsächlich weil die dienstliche Benützung zu großen Unbequemlichkeiten für den Zeitlofser SchloBbesitzer führte und bei der gesteigerten Inanspruchnahme des Archivs zu wirklichen Unzuträglichkeiten hätte führen müssen, denen durch den Neubau rechtzeitig vorgebeugt worden ist. Es hat sich schon jetzt gezeigt, daB die Verlegung im dienstlichen Interesse und in dem der Miteigentümer von großem Vorteil war.

Diese Verlegung aus dem Schlosse (wo das Archiv etwa seit dessen Erbauung im Jahre 1787 untergebracht gewesen war) erfolgte erst ein halbes Jahr nach Fertigstellung im Oktober 1912.

Das Archiv enthält für die Familie äußerst wertvolle Urkunden, Abschriften und Akten, die namentlich die Geschichte des Besitzes und seiner Rechtsverhältnisse (seit dem 15. Jahrhundert ziemlich lückenlos) verfolgen lassen. Ältere Kaiserurkunden als aus dem Anfange des 18. Jahrhunderts sind im Original meines $W$ issens nicht vorhanden - die mir bekannte älteste Original-Urkunde überhaupt ist von ,dem nehsten montag in der heiligen kruczelvochen" 1414 datiert. Abschriften von Urkunden reichen bis etwa 1136 zurück. Belehnungsurkunden von Rieneck, Mainz, Sachsen, Henneberg, Fulda, Würzburg und in neuerer Zeit auch Bayern sind in Original und Abschrift vielfach - von den letzten Jahrhunderten ziemlich lückenlos erhalten, wie auch Burgfrieden aus dem 14. und Anfang des 15. Jahrhunderts.

Die Erhaltung dieses reichen archivalischen Schatzes - das Repertorium weist über 3000 Nummern auf - ist glücklichen Umständen und der Fürsorge der Familie zu verdanken.

$\mathrm{Zu}$ den glücklichen Umständen muß man in diesem Falle die großen Prozesse der Familie rechnen, z. B. den $31 / 2$ Jahrhunderte währenden mit der Gemeinde Burgsinn, den etwa 200jährigen mit der Universität Würzburg, zu schweigen von den 100- und den 50 jährigen, die namentlich im 18. und Anfang des 19. Jahrhunderts zur Wiedergewinnung unrechtmäßig verkauften 


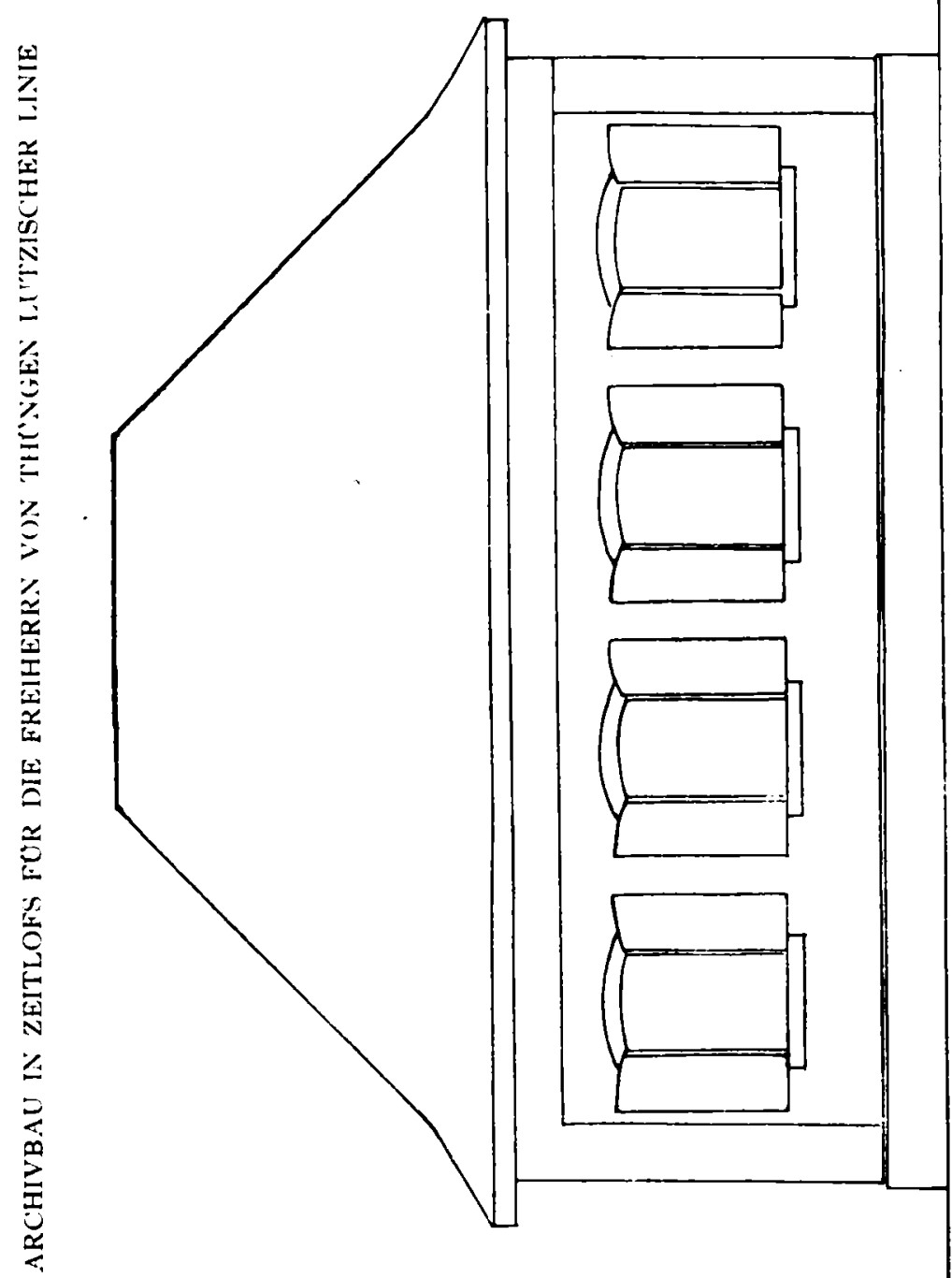




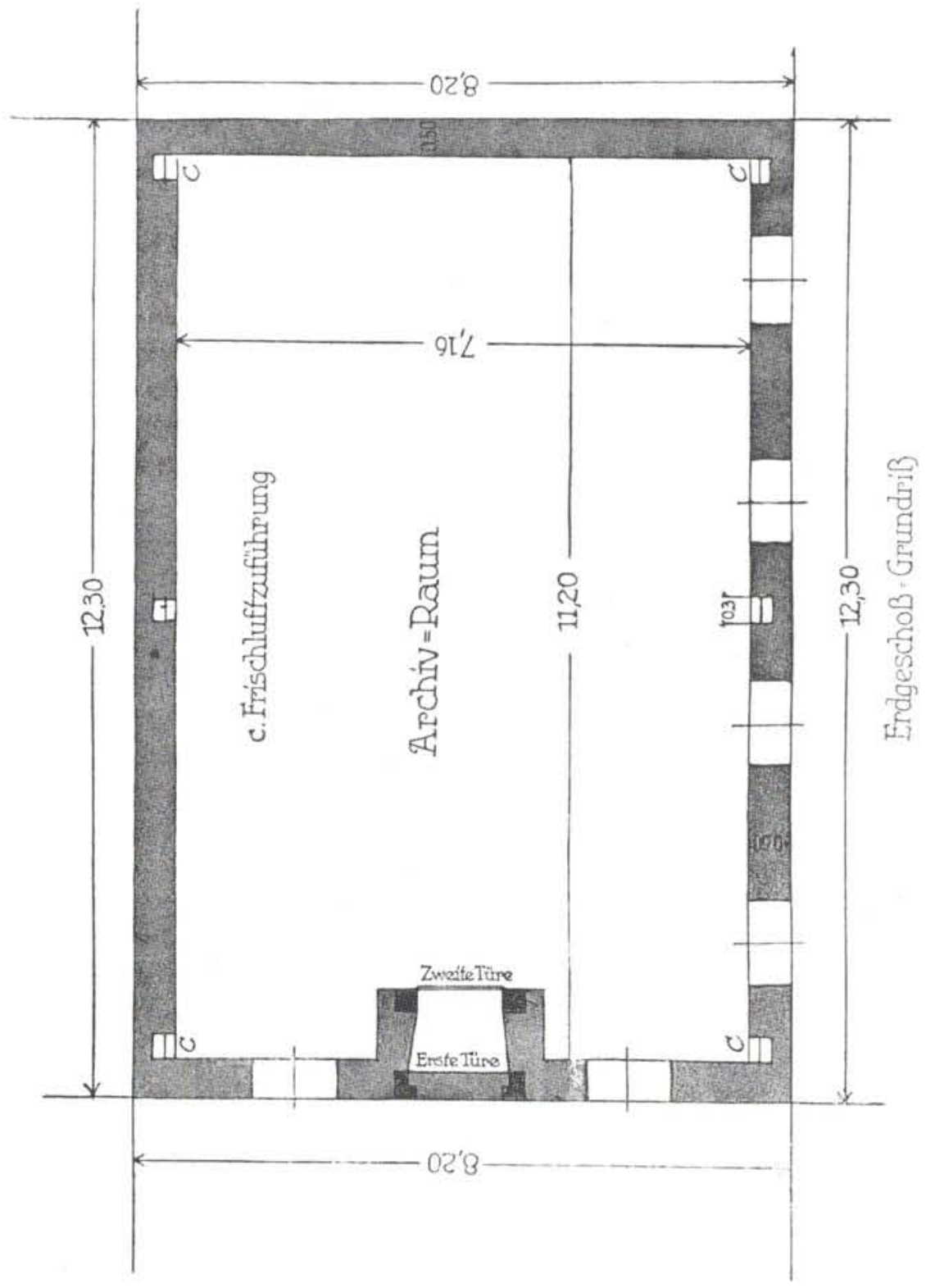


oder gewaltsam abgenommenen Besitzes mit Lehenshöfen und Regierungen vor Reichskammergericht, Reichshofrat und verschiedenen Lehenshöfen vielfach mit glücklichem Ausgang gespielt haben. Auch die oft durch mehrere Generationen sich hinziehenden Erbstreitigkeiten sind hierher zu zählen, da sie den Wert des Urkundenmaterials wohl vor Augen zu führen geeignet waren.

Aber auch ohne Prozesse hat die Familie ihre Urkunden zu erhalten gesucht. So brachten die Brüder Karl und Contz von Thüngen am 14. Mai 1437 ihre Urkunden in zwei Truhen verpackt nach der Reichsstadt Schweinfurt in Sicherheit vor dem gegen den ReuBenberg heranziehenden Kriegswetter, das am 25. September 1438 mit der siegreichen Abwehr des bischöflich würzburgischen Heeres ruhmvoll für die Familie endigte.

Hierher ist auch die erfolgreiche Verteidigung des Sodenberges im Bauernkrieg 1525 zu rechnen. Mit der Erhaltung dieses einzigen Schlosses in ganz Franken (auBer der bischöflichen Residenz „Unser lieben Frauenberg“) gegen die Bauern war auch die Erhaltung des dort zahlreich vorhandenen und dorthin geflüchteten Urkundenmaterials der Familien Thüngen und Hutten erfolgt.

Auch bei den verschiedenen Fluchtreisen vor Schweden oder Kaiserlichen im Dreißigjährigen Krieg wurden die Urkunden nicht vergessen, und Neidhard von Thüngen - sonst kein Mehrer des Besitzes! - - beklagte sich bitter im September 1633, daß ihm Urkunden ,wider alles Vermeinen bei vergangener römischer Occupation auf dem Amtshaus Homberg" ( $=$ Homburg an der Wern) verworfen worden seien.

Die Gemcinschaftlichkeit des Besitzes hatte sehr vielfach auch die Urkunden der Andreasischen Linie sei es im Original, sei es in Abschrift in den Besitz der Lutzischen Linie gebracht, so daB der Bestand des Archivs -- wenn man in früheren Zeiten diese Bezeichnung anwenden will - mit der Zeit durch wichtiges Material aus der anderen Hauptlinie der Familie bereichert worden ist. Aber eine Vereinigung fast sämtlicher Thüngenschen Urkunden und Akten in e i n e m Archiv fand wohl erst statt, als die Lutzische Linie auf zwei Augen gestellt war (Philipp Christoph Dietrich von Thüngen, Ritterhauptmann, 1696-1780). Bis dahin waren die Urkunden nach dem Besitz auf die ein- 
zelnen Linien, Zweige und sogar Personen verteilt, wie es ja eine oft wiederkehrende Bestimmung in den Erbteilungsverträgen war, wo die Urkunden aufgehoben und wie sie den verschiedenen Eigentümern zugänglich gemacht werden sollten.

ARLHIIB.IT ZEITLOFS

Querschnitt

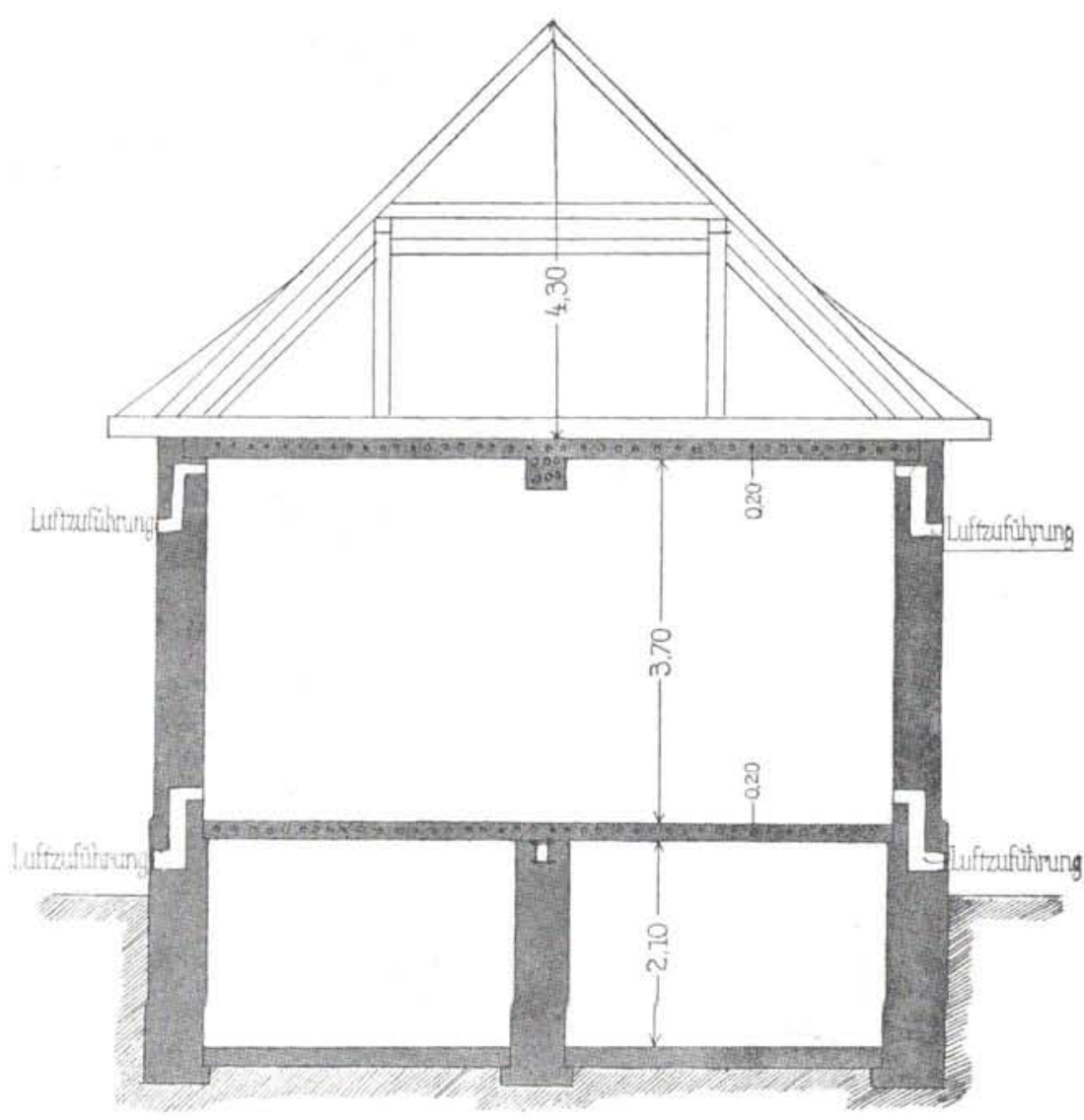

Maßstab 1:100

Zu Philipp Christoph Dietrichs Zeiten wird von einem Brand berichtet, der die "Repositur" in Unordnung gebracht habe; seine Söhne mußten vor der Bayrisch-Hessischen „Occupation“ (1803 04) ihre "Akten" aus Zeitlofs retten - wohin ist nicht überliefert … und hatten es erst anfangs 1807 gewagt, sie teil- 
weise wieder zurückbringen zu lassen. Zu dieser Zeit war also Zeitlofs bereits zum Hauptarchiv der Familie geworden, und nur einiges auf den Ort Thüngen und Umgebung Bezügliche mag dortselbst untergebracht gew'esen sein. So bildete sich das Archiv seit Ende des 18. Jahrhunderts zu dem aus, was es jetzt ist: Das Archiv der Freiherren von Thüngen Lutzischer Linie.

Seit der ersten Hälfte des 19. Jahrhunderts entstand dann auch das Bedürfnis zur Regelung der Benützung, die bei einem Kondominat, wo jeder Miteigentümer auch Mitherr des Archivs ist, unentbehrlich ist.

Die erste derartige Regelung brachte das Familienstatut vom Jahre 1862, die hauptsächlich aus der Sorge hervorgegangen war, daB einzelne Eigentümer ihr persönliches Recht, namentlich bei miBliebigen Akten, über das Recht der Gesamtfamilie setzen könnten. Sie enthielt daher in der Hauptsache die folgenden Bestimmungen: Das Archiv kann und darf nur von einem der beiden Familienbevollmächtigten in Gemeinschaft mit dem Rentamtmann der Familie geöffnet werden. Nur in ihrer Gegenwart können die übrigen Familienglieder das Archiv betreten. Archivalien werden auch an Miteigentümer nur gegen schriftliche Bescheinigung ausgegeben.

Aber die damaligen Archivherren schützten ihr Eigentum nicht nur vor Mißbrauch, sie erkannten auch die Vorteile eines geregelten Gebrauchs und ließen eine Neuordnung vornehmen, die dringend notwendig geworden war, da die ungeschulten Kräfte der Bewältigung des immer zunehmenden Materials nicht mehr gewachsen waren. Diese Aufgabe löste der damalige Reichsarchiv-Assessor Dr. Häntle mit Geschick und Verständnis. Er ordnete vom 26. Juli bis 29. August 1871 die Archivalien an Ort und Stelle und verfaßte dann ein Repertorium (mit Sach-, Orts- und Personenregister), das noch heute allen billigerweise zu stellenden Anforderungen entspricht und eine sachliche Ausbeutung des Archivs ermöglicht.

Die Bestimmungen über die Archivbenützung erwiesen sich aber bei gröBerer Ausdehnung der Familie und für eine fruchtbare Benützung des Archivs durch die Eigentümer als etwas zu enge. Es wurde deshalb am 12. August 1912 für das neue Archiv eine neue Ordnung (s. Anhang) vereinbart, die bei Wah- 
rung der Rechte der Eigentümer an ihr Archiv dieses selbst doch möglichst gegen allenfallsigen Mißbrauch der einzelnen zu wahren sucht und der sonstigen Verfassung der Familie angepaBt ist.

N a u m b u rg a. d. Saale, 9. Dezember 1913.

Frhr.v. Thüngen.

\section{Anhang.}

Die unterzeichneten Freiherren von Thüngen Lutzischer Linie als derzeitige Gesamtheit der stimmberechtigten Familienglieder haben auf Grund des $\S 10$ Ziffer $7 \mathrm{a}$ ihres Familienstatuts vom Jahre 1862 die folgende Ordnung eingeführt, die vom Tage des Bezuges ihres neu erbauten Archivs in Zeitlofs in Geltung tritt.

$$
\text { A r c h i v-Ordnung. }
$$

1. Das Freiherrlich von Thüngensche Familien-Archiv zu Zeitlofs steht unter der Oberaufsicht der Familienbevollmächtigten, denen der Rentamtmann beigegeben ist. Statt des Rentamtmanns können die Familienbevollmächtigten einen anderen $\mathrm{Be}-$ amten aushilfsweise bestimmen.

2. Es ist verboten, im Archiv zu rauchen, es mit Licht zu betreten oder darin Siegelungen vorzunehmen.

3. Das Archiv steht unter doppeltem VerschluB. Den Schlüssel zu einem Schloß hat der Rentamtmann, zum anderen Schloß erhält jeder volljährige Miteigentümer einen Schlüssel.

4. Die Familienbevollmächtigten müssen zu jeder Zeit von den Schlüsselbesitzern schriftlich vom Aufbewahrungsort der Schlüssel unterrichtet sein. Die von jedem Schlüsselbesitzer zu dem Zweck an die Familienbevollmächtigten erstmalig zu erstattenden Anzeigen sind im Rentamte niederzulegen. Die Familienbevollmächtigten haben die Pflicht, jährlich einmal sich den Aufbewahrungsort der Schlüssel aufs neue bescheinigen zu lassen. Sie haben das Recht, die Schlüssel behufs Kontrolle unvorbereitet einzuverlangen, oder die Änderung des Aufbewahrungsortes seitens der Besitzer zu verlangen, wenn der angezeigte offensichtlich nicht sicher genug erscheint.

5. Kommt der Schlüsselbesitzer dieser Aufforderung nicht nach, schickt er den Schlüssel auf Aufforderung nicht ein, so 
haben die Familienbevollmächtigten das Recht - bei Verlust eines Schlüssels haben sie die Pflicht - , das Schloß auf Kosten des schuldigen Familiengliedes abändern zu lassen. Der Familie ist dies anzuzeigen. Sie hat dann das Recht, mit zwei Drittel Mehrheit dem bisherigen Inhaber die Schlüsselführung abzusprechen. Überzählig gewordene Schlüssel werden im Archiv selbst - versiegelt von den beiden Familienbevollmächtigten und dem Rentamtmann - niedergelegt. Mehr als einen Schlüssel darf kein Familienglied besitzen.

6. Die Familienbevollmächtigten und der Rentamtmann haben im Erbfall für sofortige Sicherstellung der Schlüssel zu sorgen.

7. Das Archiv darf nur von den auf dem Rentamt angegebenen Schlüsselbesitzern im Verein mit dem Rentamtmann oder dessen Stellvertreter geöffnet werden. Die Schlüsselübergabe eines Besitzers an den Rentamtmann oder umgekehrt behufs Öffnung des Archivs ist verboten.

8. Der Rentamtmann oder dessen Stellvertreter ist im allgemeinen verpflichtet, während der Anwesenheit eines Familiengliedes im Archiv zu verweilen, und darf von diesem unter keinem Vorwande aus dem Archiv entfernt werden. In Ausnahmefällen kann der Rentamtmann schriftlich von den Familienbevollmächtigten von der Verpflichtung der immerwährenden Anwesenheit entbunden werden, nicht aber von der der Anwesenheit bei Öffnung und Schließen des Archivs. Der Rentamtmann kann in allen Fällen durch einen arderen, von den Familienbevollmächtigten bestimmten Beamten vertreten werden.

9. Für die Ordnung im Archiv ist der Rentamtmann unter (rormular 4$)$ ) der Oberaufsicht der Familienbevollmächtigten verantwortlich. Die Familienbevollmächtigten haben sich möglichst oft durch Stichproben und alljährlich einmal durch gemeinschaftliches Nachsehen davon zu überzeugen, daß die Akten und Urkunden unversehrt an ihrem Platze liegen. Protokolle über die jährliche Revision sind aufzunehmen.

Es dürfen daher Akten und Urkunden von niemand ohne Vorwissen des Rentamtmanns oder ohne ihm davon genaue Mitteilung zu machen von ihrem ordnungsmäßigen Platz auch nur zeitweilig entfernt werden.

10. Nur der Rentamtmann oder dessen Stellvertreter ist 
berechtigt, Akten und Urkunden aus dem Archiv an Familienglieder gegen Bescheinigung auszuhändigen. Diese hat seitens des Benützers eine ausdrückliche Anerkennung der Ziffern 11 mit 14 dieser Ordnung zu enthalten. Dieser Bestimmung sind auch die Familienbevollmächtigten unterworfen.

Es ist dem Rentantmann verboten, an andere als Familienglieder Akten und Urkunden auszuhändigen. Hierzu bedarf es, wie z. B. bei Prozessen, der schriftlichen Genehmigung der beiden Familienbevollmächtigten.

11. Allen Benützern von Akten oder Urkunden - auch den Aufsichtsorganen des Archivs - ist verboten, Anmerkungen oder Zeichen irgendwelcher Art darauf anzubringen. Der Rentamtmann hat bei der Rückgabe von Schriftstücken sie daraufhin zu prüfen. Finden sich hierbei Änderungen usw., so hat er dies unverzüglich den beiden Familienbevollmächtigten gleichzeitig in doppelter Ausfertigung auch dann schriftlich zu melden, wenn einer der beiden der Benützer war. Die Familienbevollmächtigten sind verpflichtet, die Meldung der Familie bekannt zu geben.

12. Akten und Urkunden, welche an Familienglieder zur Benützung außerhalb des Archivs gegeben werden, sind von diesen auf das sorgfältigste aufzubewahren. Für Verlust und Beschädigung sind sie haftbar. Findet eine Einigung auf gütlichem Wege nicht statt, so ist die Höhe der Entschädigung von den übrigen Mitbesitzern auf Vorschlag der Familienbevollmächtigten festzusetzen. Bei wichtigen Urkunden kann ein staatlicher Archivbeamter gutachtlich vernommen werden. Die Kosten des Verfahrens trägt der den Verlust Verschuldende.

13. Bei Verfehlungen gegen Ziffer 11 und 12 kann bis zum ersten Wiederholungsfalle, bei öfteren muß dem Betreffenden das Recht abgesprochen werden, Akten und Urkunden anders als im Beisein des Beamten oder eines Familienbevollmächtigten im Archiv einzusehen.

14. Sind Akten und Urkunden, die einem Familiengliede zur Benützung übergeben waren, für einen ProzeB oder die Familienverwaltung nötig, so sind sie auf Verlangen der Familienbevollmächtigten sofort zurückzugeben. Wünscht sie ein anderes Familienglied zu privater Benutzung, so sind sie ihm spätestens vier Wochen nach dem geäußerten Verlangen auszu- 
händigen. Sind Akten länger als ein halbes Jahr in Händen eines Familiengliedes, so können die Familienbevollmächtigten sie zur Kontrolle (auf kurze Zeit, wenn das Familienglied sie noch gebraucht) zurückverlangen. Statt des Einsendens der Akten können sich die Familienbevollmächtigten, wenn ihnen die Lage danach zu sein scheint, auch mit einer Bescheinigung des Benützers begnügen, daB die Akten usw. noch unversehrt in seinen Händen sind.

Wird die Zurückgabe der Akten verweigert, so können die Familienbevollmächtigten, außer den sonst nötigen Schritten zur Wiedererlangung, bei der Familie beantragen, daB den betreffenden Herren in Zukunft keine Akten mehr ausgehändigt werden. Die einfache Mehrheitsentscheidung der Familie hierüber ist rechtsgültig.

15. Abschriften von Akten usw. sind jedem Mitbesitzer auf Verlangen auf seine Kosten zu liefern. Für die Anfertigung haben die Familienbevollmächtigten zu sorgen.

16. Jeder Empfänger eines Schlüssels hat sich durch Unterschrift zu verpflichten, diese Archivordnung in allen Punkten zu befolgen und aufrecht zu erhalten.

17. Mit Einführung dieser Archivordnung tritt $\$ 11$ Ziff. 2 Absatz 3 des Familienstatuts von: ,Insbesondere ist den Bevollmächtigten die Aufsicht über das Familien-Archiv zu Zeitlofs übertragen" usw. bis : „... für deren Anfertigung die Bevollmächtigten zu sorgen haben" außer Kraft. Die Anrufung eines Schiedsgerichts ist durch die Bestimmungen der vorstehenden Ziffern 5 und 14 nicht berührt.

Roßbach, Weißenbach, Würzburg, Thüngen, Heilsberg, Zeitlofs und Bamberg am 25. August 1912.

Carl Freiherr von Thüngen.

Reinhard Freiherr von Thüngen.

Wilhelm Freiherr von Thüngen.

Hans Freiherr von Thüngen.

Rudolf Freiherr von Thüngen.

Conrad Freiherr von Thüngen.

Hildolf Freiherr von Thüngen. 